\title{
Modifications of the Third Zadeh's intuitionistic fuzzy implication
}

\author{
Krassimir T. Atanassov ${ }^{1,2}$ and Nora Angelova ${ }^{3}$ \\ ${ }^{1}$ Dept. of Bioinformatics and Mathematical Modelling, \\ Institute of Biophysics and Biomedical Engineering, \\ Bulgarian Academy of Sciences \\ 105 Acad. G. Bonchev Str., 1113 Sofia, Bulgaria \\ ${ }^{2}$ Intelligent Systems Laboratory \\ Prof. Dr. Asen Zlatarov University, 8010 Burgas, Bulgaria \\ e-mail: krat@bas.bg \\ ${ }^{3}$ Faculty of Mathematics and Informatics, Sofia University \\ 5 James Bourchier Blvd., 1164 Sofia, Bulgaria \\ e-mail: noraalfmi.uni-sofia.bg
}

\begin{abstract}
In [24], G. Klir and B. Yuan named after L. Zadeh the implication $p \rightarrow q=$ $\max (1-p, \min (p, q))$. In a series of papers, the author introduced two intuitionistic fuzzy forms of Zadeh's implication and their basic properties have been studied. In the present paper, a new (third) intuitionistic fuzzy form of Zadeh's implication is given and some of its properties are studied.
\end{abstract}

Keywords: Intuitionistic fuzzy implication, Intuitionistic fuzzy set, Zadeh's fuzzy implication. 2020 Mathematics Subject Classification: 03E72.

\section{Introduction}

Implication is one of the basic operations in logic, and in intuitionistic fuzzy logic it has become a special object of investigation. Due to its definition, where the second degree - of non-membership, non-validity, etc. - has been introduced, intuitionistic fuzzy sets allow working with not just one, but multiple differently defined intuitionistic fuzzy implications. The extensive research 
of intuitionistic fuzzy implications, as well as of intuitionistic fuzzy negations, started in the beginning of the 2000s and it has lead to the definition of more than 190 different implications.

In $[8,9]$, the first implication has number 1 and in [9] the second one is numbered as 166-th. Notably, in [8] there are definitions of 138 implications, while in [9] they are 185. Among the new 47 implications, published after 2012, 22 of them were introduced by L. Atanassova in $[13,14,15,16,17,18]$ and three by P. Dworniczak in $[20,21,22]$. All other intuitionistic fuzzy implications are introduced by the author, in some cases in a collaboration with B. Riečan, E. Szmidt, J. Kacprzyk, N. Angelova and V. Atanassova. The 190 implications from [27] generate 55 different negations.

We must mention that we ascertained that intuitionistic fuzzy implication $\rightarrow_{191}$ introduced in [10] coincided with implication $\rightarrow_{24}$ and therefore, it was omitted in the full list of the intuitionistic fuzzy implications. On the other hand, in [11], we gave an intuitionistic fuzzy form of the Goguen's implication and now, it will obtain the sequential number 191, while the Third Zadeh's intuitionistic fuzzy implication, which was recently introduced in [12] obtains the sequential number 192 .

The Third Zadeh's intuitionistic fuzzy implication is based on Zadeh's fuzzy implication that has the form as follows

$$
p \rightarrow q=\max (1-p, \min (p, q))
$$

(see, e.g., $[19,24])$ and on the two Zadeh's intuitionistic fuzzy implications introduced by the author (see, [8]).

Here, following and combining ideas from [6, 23], we will construct six new implications, related to the Third Zadeh's intuitionistic fuzzy implication, and in the present paper they are assigned the sequential numbers 193-rd to 198-th, respectively.

Let a set $E$ be fixed. The Intuitionistic Fuzzy Set (IFS; see [4, 8]) $A$ in $E$ is defined by:

$$
A=\left\{\left\langle x, \mu_{A}(x), \nu_{A}(x)\right\rangle \mid x \in E\right\},
$$

where functions $\mu_{A}: E \rightarrow[0,1]$ and $\nu_{A}: E \rightarrow[0,1]$ define the degree of membership and the degree of non-membership of the element $x \in E$, respectively, and for every $x \in E$ :

$$
0 \leq \mu_{A}(x)+\nu_{A}(x) \leq 1
$$

The three Zadeh's intuitionistic fuzzy implications have the forms:

$$
A \rightarrow_{1} B=\left\{\left\langle x, \max \left(\nu_{A}(x), \min \left(\mu_{A}(x), \mu_{B}(x)\right)\right), \min \left(\mu_{A}(x), \nu_{B}(x)\right)\right\rangle \mid x \in E\right\} .
$$

(see $[5,8,9])$,

$$
\begin{aligned}
A \rightarrow_{166} B=\{\langle x, & \max \left(\nu_{A}(x), \min \left(\mu_{A}(x), \mu_{B}(x)\right)\right), \\
& \left.\left.\min \left(\mu_{A}(x), \max \left(\nu_{A}(x), \nu_{B}(x)\right)\right)\right\rangle \mid x \in E\right\}
\end{aligned}
$$

$($ see $[7,9])$ and

$$
\begin{aligned}
A \rightarrow_{192} B=\left\{\left\langlex, \max \left(\mu_{B}(x), \min \left(\nu_{A}(x), \nu_{B}(x)\right)\right),\right.\right. & \\
& \left.\left.\min \left(\nu_{B}(x), \max \left(\mu_{A}(x), \mu_{B}(x)\right)\right)\right\rangle \mid x \in E\right\} .
\end{aligned}
$$

(see [12]). 


\section{Preliminary results}

In the beginning, the necessary concepts from intuitionistic fuzzy set theory will be given, following [8].

Let for every $x \in E$ :

$$
\pi_{A}(x)=1-\mu_{A}(x)-\nu_{A}(x) .
$$

Therefore, function $\pi$ determines the degree of uncertainty.

Let us define the empty IFS, the totally uncertain IFS, and the unit IFS (see [4, 8]) by:

$$
\begin{aligned}
& O^{*}=\{\langle x, 0,1\rangle \mid x \in E\}, \\
& U^{*}=\{\langle x, 0,0\rangle \mid x \in E\}, \\
& E^{*}=\{\langle x, 1,0\rangle \mid x \in E\} .
\end{aligned}
$$

The geometrical interpretation of an element $x \in E$ with degrees $\mu_{A}(x)$ and $\nu_{A}(x)$ are shown on Fig. 1 (see $[4,8])$.

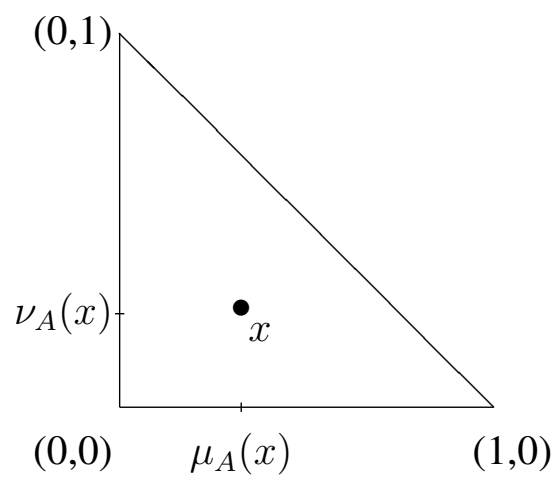

Figure 1. The geometrical interpretation of an element $x \in E$

An IFS $A$ is called Intuitionistic Fuzzy Tautological Set (IFTS) if and only if (iff) for every $x \in E$, it holds that $\mu_{A}(x) \geq \nu_{A}(x)$, and it is a Tautological Set iff for every $x \in E: \mu_{A}(X)=1$, $\nu_{A}(x)=0$.

For two IFSs $A$ and $B$ :

$$
A \subseteq B \text { iff }(\forall x \in E)\left(\mu_{A}(x) \leq \mu_{B}(x) \& \nu_{A}(x) \geq \nu_{B}(x)\right)
$$

Therefore, for each IFS $A$ :

$$
A \rightarrow_{166} B \subseteq A \rightarrow_{1} B .
$$

Over a fixed IFS $A$ standard and extended modal operators are defined (see [8]). Here, we use only two of them:

$$
\begin{aligned}
& \square A=\left\{\left\langle x, \mu_{A}(x), 1-\mu_{A}(x)\right\rangle \mid x \in E\right\}, \\
& \diamond A=\left\{\left\langle x, 1-\nu_{A}(x), \nu_{A}(x)\right\rangle \mid x \in E\right\} .
\end{aligned}
$$




\section{Main results}

In [23], if $\supset$ is a fixed implication, the following new modal implication can be introduced:

$$
p \rightarrow q=\square(p \supset q)
$$

where $p$ and $q$ are propositional variables. Having the Third Zadeh's intuitionistic fuzzy implication $\left(\rightarrow_{192}\right)$ and following [6], we will construct six new implications.

$$
\begin{aligned}
A \rightarrow_{193} B= & \square\left(A \rightarrow_{192} B\right) \\
= & \square\left(\left\{\left\langle x, \mu_{A}(x), \nu_{A}(x)\right\rangle \mid x \in E\right\} \rightarrow_{Z}\left\{\left\langle x, \mu_{B}(x), \nu_{B}(x)\right\rangle \mid x \in E\right\}\right) \\
= & \square\left\{\left\langle x, \max \left(\mu_{B}(x), \min \left(\nu_{A}(x), \nu_{B}(x)\right)\right), \min \left(\nu_{B}(x), \max \left(\mu_{A}(x), \mu_{B}(x)\right)\right)\right\rangle \mid x \in E\right\} \\
= & \left\{\left\langle x, \max \left(\mu_{B}(x), \min \left(\nu_{A}(x), \nu_{B}(x)\right)\right), 1-\max \left(\mu_{B}(x), \min \left(\nu_{A}(x), \nu_{B}(x)\right)\right)\right\rangle \mid x \in E\right\} . \\
A \rightarrow_{194} B= & \diamond\left(A \rightarrow_{192} B\right) \\
= & \diamond\left(\left\{\left\langle x, \mu_{A}(x), \nu_{A}(x)\right\rangle \mid x \in E\right\} \rightarrow_{Z}\left\{\left\langle x, \mu_{B}(x), \nu_{B}(x)\right\rangle \mid x \in E\right\}\right) \\
= & \diamond\left\{\left\langle x, \max \left(\mu_{B}(x), \min \left(\nu_{A}(x), \nu_{B}(x)\right)\right), \min \left(\nu_{B}(x), \max \left(\mu_{A}(x), \mu_{B}(x)\right)\right)\right\rangle \mid x \in E\right\} \\
= & \left\{\left\langle x, 1-\min \left(\nu_{B}(x), \max \left(\mu_{A}(x), \mu_{B}(x)\right)\right), \min \left(\nu_{B}(x), \max \left(\mu_{A}(x), \mu_{B}(x)\right)\right)\right\rangle \mid x \in E\right\} . \\
A \rightarrow{ }_{195} B= & \square A \rightarrow{ }_{192} B B \\
= & \square\left\{\left\langle x, \mu_{A}(x), \nu_{A}(x)\right\rangle \mid x \in E\right\} \rightarrow_{Z} \square\left\{\left\langle x, \mu_{B}(x), \nu_{B}(x)\right\rangle \mid x \in E\right\} \\
= & \left\{\left\langle x, \mu_{A}(x), 1-\mu_{A}(x)\right\rangle \mid x \in E\right\} \rightarrow_{Z}\left\{\left\langle x, \mu_{B}(x), 1-\mu_{B}(x)\right\rangle \mid x \in E\right\} \\
= & \left\{\left\langlex, \max \left(\mu_{B}(x), \min \left(1-\mu_{A}(x), 1-\mu_{B}(x)\right)\right),\right.\right. \\
& \left.\left.\quad \min \left(1-\mu_{B}(x), \max \left(\mu_{A}(x), \mu_{B}(x)\right)\right)\right\rangle \mid x \in E\right\} \\
= & \left\{\left\langlex, \max \left(\mu_{B}(x), 1-\max \left(\mu_{A}(x), \mu_{B}(x)\right)\right),\right.\right. \\
& \left.\left.\quad \min \left(1-\mu_{B}(x), \max \left(\mu_{A}(x), \mu_{B}(x)\right)\right)\right\rangle \mid x \in E\right\} \\
= & \left\{\left\langlex, \max \left(\mu_{B}(x), 1-\max \left(\mu_{A}(x), \mu_{B}(x)\right)\right),\right.\right. \\
& \left.\left.1-\max \left(\mu_{B}(x), 1-\max \left(\mu_{A}(x), \mu_{B}(x)\right)\right)\right\rangle \mid x \in E\right\} .
\end{aligned}
$$

$$
\begin{aligned}
A \rightarrow_{196} B= & \square A \rightarrow_{192} \diamond B \\
= & \square\left\{\left\langle x, \mu_{A}(x), \nu_{A}(x)\right\rangle \mid x \in E\right\} \rightarrow_{Z} \diamond\left\{\left\langle x, \mu_{B}(x), \nu_{B}(x)\right\rangle \mid x \in E\right\} \\
= & \left\{\left\langle x, \mu_{A}(x), 1-\mu_{A}(x)\right\rangle \mid x \in E\right\} \rightarrow_{Z}\left\{\left\langle x, 1-\nu_{B}(x), \nu_{B}(x)\right\rangle \mid x \in E\right\} \\
= & \left\{\left\langlex, \max \left(1-\nu_{B}(x), \min \left(1-\mu_{A}(x), \nu_{B}(x)\right)\right),\right.\right. \\
& \left.\left.\quad \min \left(\nu_{B}(x), \max \left(\mu_{A}(x), 1-\nu_{B}(x)\right)\right)\right\rangle \mid x \in E\right\} \\
= & \left\{\left\langlex, \max \left(1-\nu_{B}(x), 1-\max \left(\mu_{A}(x), 1-\nu_{B}(x)\right)\right),\right.\right. \\
& \left.\left.\quad \min \left(\nu_{B}(x), \max \left(\mu_{A}(x), 1-\nu_{B}(x)\right)\right)\right\rangle \mid x \in E\right\} \\
= & \left\{\left\langlex, 1-\min \left(\nu_{B}(x), \max \left(\mu_{A}(x), 1-\nu_{B}(x)\right)\right),\right.\right. \\
& \left.\quad \min \left(\nu_{B}(x), \max \left(\mu_{A}(x), 1-\nu_{B}(x)\right)\right\rangle \mid x \in E\right\} .
\end{aligned}
$$




$$
\begin{aligned}
A \rightarrow_{197} B= & \diamond A \rightarrow_{192} \square B \\
= & \diamond\left\{\left\langle x, \mu_{A}(x), \nu_{A}(x)\right\rangle \mid x \in E\right\} \rightarrow_{Z} \square\left\{\left\langle x, \mu_{B}(x), \nu_{B}(x)\right\rangle \mid x \in E\right\} \\
= & \left\{\left\langle x, 1-\nu_{A}(x), \nu_{A}(x)\right\rangle \mid x \in E\right\} \rightarrow_{Z}\left\{\left\langle x, \mu_{B}(x), 1-\mu_{B}(x)\right\rangle \mid x \in E\right\} \\
= & \left\{\left\langlex, \max \left(\mu_{B}(x), \min \left(\nu_{A}(x), 1-\mu_{B}(x)\right)\right),\right.\right. \\
& \left.\left.\quad \min \left(1-\mu_{B}(x), \max \left(1-\nu_{A}(x), \mu_{B}(x)\right)\right)\right\rangle \mid x \in E\right\} \\
= & \left\{\left\langlex, \max \left(\mu_{B}(x), \min \left(\nu_{A}(x), 1-\mu_{B}(x)\right)\right),\right.\right. \\
& \left.\left.\quad \min \left(1-\mu_{B}(x), 1-\min \left(\nu_{A}(x), 1-\mu_{B}(x)\right)\right)\right\rangle \mid x \in E\right\} \\
= & \left\{\left\langlex, \max \left(\mu_{B}(x), \min \left(\nu_{A}(x), 1-\mu_{B}(x)\right)\right),\right.\right. \\
& \left.\left.\quad 1-\max \left(\mu_{B}(x), \min \left(\nu_{A}(x), 1-\mu_{B}(x)\right)\right)\right\rangle \mid x \in E\right\} . \\
A \rightarrow{ }_{198} B= & \diamond A \rightarrow \rightarrow_{192} \diamond B \\
= & \diamond\left\{\left\langle x, \mu_{A}(x), \nu_{A}(x)\right\rangle \mid x \in E\right\} \rightarrow_{Z} \diamond\left\{\left\langle x, \mu_{B}(x), \nu_{B}(x)\right\rangle \mid x \in E\right\} \\
= & \left\{\left\langle x, 1-\nu_{A}(x), \nu_{A}(x)\right\rangle \mid x \in E\right\} \rightarrow_{Z}\left\{\left\langle x, 1-\nu_{B}(x), \nu_{B}(x)\right\rangle \mid x \in E\right\} \\
= & \left\{\left\langlex, \max \left(1-\nu_{B}(x), \min \left(\nu_{A}(x), \nu_{B}(x)\right)\right),\right.\right. \\
& \left.\left.\quad \min \left(\nu_{B}(x), \max \left(1-\nu_{A}(x), 1-\nu_{B}(x)\right)\right)\right\rangle \mid x \in E\right\} \\
= & \left\{\left\langlex, \max \left(1-\nu_{B}(x), \min \left(\nu_{A}(x), \nu_{B}(x)\right)\right),\right.\right. \\
& \left.\left.\quad \min \left(\nu_{B}(x), 1-\min \left(\nu_{A}(x), \nu_{B}(x)\right)\right)\right\rangle \mid x \in E\right\} \\
= & \left\{\left\langlex, \max \left(1-\nu_{B}(x), \min \left(\nu_{A}(x), \nu_{B}(x)\right)\right),\right.\right. \\
& \left.\left.1-\max \left(1-\nu_{B}(x), \min \left(\nu_{A}(x), \nu_{B}(x)\right)\right)\right\rangle \mid x \in E\right\} . \\
&
\end{aligned}
$$

First, we check that the definitions are correct.

For implication $\rightarrow_{193}$, obviously,

$$
\begin{aligned}
& 0 \leq \max \left(\mu_{B}(x), \min \left(\nu_{A}(x), \nu_{B}(x)\right)\right) \leq 1, \\
& 0 \leq 1-\max \left(\mu_{B}(x), \min \left(\nu_{A}(x), \nu_{B}(x)\right)\right) \leq 1
\end{aligned}
$$

and

$$
\max \left(\mu_{B}(x), \min \left(\nu_{A}(x), \nu_{B}(x)\right)\right)+1-\max \left(\mu_{B}(x), \min \left(\nu_{A}(x), \nu_{B}(x)\right)\right)=1 .
$$

The same is valid for the rest of the implications.

Following $[4,8]$, we define the classical intuitionistic fuzzy negation by:

$$
\neg A=A \rightarrow_{193} O^{*}=\left\{\left\langle x, \nu_{A}(x), \mu_{A}(x)\right\rangle \mid x \in E\right\} .
$$

We can check directly that

$$
\begin{aligned}
& A \rightarrow_{193} U^{*}=\left\{\left\langle x, \max \left(0, \min \left(\nu_{A}(x), 0\right)\right), 1-\max \left(0, \min \left(\nu_{A}(x), 0\right)\right)\right\rangle \mid x \in E\right\}=O^{*}, \\
& A \rightarrow_{193} E^{*}=\left\{\left\langle x, \max \left(1, \min \left(\nu_{A}(x), 0\right)\right), 1-\max \left(1, \min \left(\nu_{A}(x), 0\right)\right)\right\rangle \mid x \in E\right\}=E^{*}, \\
& A \rightarrow_{194} U^{*}=\left\{\left\langle x, 1-\min \left(0, \max \left(\mu_{A}(x), 0\right)\right), \min \left(0, \max \left(\mu_{A}(x), 0\right)\right)\right\rangle \mid x \in E\right\}=E^{*}, \\
& A \rightarrow_{194} E^{*}=\left\{\left\langle x, 1-\min \left(0, \max \left(\mu_{A}(x), 1\right)\right), \min \left(0, \max \left(\mu_{A}(x), 1\right)\right)\right\rangle \mid x \in E\right\}=E^{*},
\end{aligned}
$$


$A \rightarrow_{195} U^{*}=\left\{\left\langle x, \max \left(0,1-\max \left(\mu_{A}(x), 0\right)\right), 1-\max \left(0,1-\max \left(\mu_{A}(x), 0\right)\right)\right\rangle \mid x \in E\right\}$

$=\left\{\left\langle x, 1-\mu_{A}(x), \mu_{A}(x)\right\rangle \mid x \in E\right\}=\diamond \neg A$,

$A \rightarrow_{195} E^{*}=\left\{\left\langle x, \max \left(1,1-\max \left(\mu_{A}(x), 1\right)\right), 1-\max \left(1,1-\max \left(\mu_{A}(x), 1\right)\right)\right\rangle \mid x \in E\right\}=E^{*}$,

$A \rightarrow{ }_{196} U^{*}=\left\{\left\langle x, 1-\min \left(0, \max \left(\mu_{A}(x), 1\right)\right), \min \left(0, \max \left(\mu_{A}(x), 1\right)\right)\right\rangle \mid x \in E\right\}=E^{*}$,

$A \rightarrow_{196} E^{*}=\left\{\left\langle x, 1-\min \left(0, \max \left(\mu_{A}(x), 1\right)\right), \min \left(0, \max \left(\mu_{A}(x), 1\right)\right)\right\rangle \mid x \in E\right\}=E^{*}$,

$A \rightarrow_{197} U^{*}=\left\{\left\langle x, \max \left(0, \min \left(\nu_{A}(x), 1\right)\right), 1-\max \left(0, \min \left(\nu_{A}(x), 1\right)\right)\right\rangle \mid x \in E\right\}$

$=\left\{\left\langle x, \nu_{A}(x), 1-\nu_{A}(x)\right\rangle \mid x \in E\right\}=\diamond A$,

$A \rightarrow_{197} E^{*}=\left\{\left\langle x, \max \left(1, \min \left(\nu_{A}(x), 0\right)\right), 1-\max \left(1, \min \left(\nu_{A}(x), 0\right)\right)\right\rangle \mid x \in E\right\}=E^{*}$,

$A \rightarrow{ }_{198} U^{*}=\left\{\left\langle x, \max \left(1, \min \left(\nu_{A}(x), 0\right)\right), 1-\max \left(1, \min \left(\nu_{A}(x), 0\right)\right)\right\rangle \mid x \in E\right\}=E^{*}$,

$A \rightarrow_{198} E^{*}=\left\{\left\langle x, \max \left(1, \min \left(\nu_{A}(x), 0\right)\right), 1-\max \left(1, \min \left(\nu_{A}(x), 0\right)\right)\right\rangle \mid x \in E\right\}=E^{*}$.

In the particular case, we have

$$
\begin{array}{lll}
O^{*} \rightarrow_{193} O^{*}=E^{*} & O^{*} \rightarrow_{194} O^{*}=E^{*} & O^{*} \rightarrow_{195} O^{*}=E^{*} \\
O^{*} \rightarrow_{193} U^{*}=O^{*} & O^{*} \rightarrow_{194} U^{*}=E^{*} & O^{*} \rightarrow_{195} U^{*}=E^{*} \\
O^{*} \rightarrow_{193} E^{*}=E^{*} & O^{*} \rightarrow_{194} E^{*}=E^{*} & O^{*} \rightarrow_{195} E^{*}=E^{*} \\
U^{*} \rightarrow_{193} O^{*}=O^{*} & U^{*} \rightarrow_{194} O^{*}=E^{*} & U^{*} \rightarrow_{195} O^{*}=E^{*} \\
U^{*} \rightarrow_{193} U^{*}=O^{*} & U^{*} \rightarrow_{194} U^{*}=E^{*} & U^{*} \rightarrow_{195} U^{*}=E^{*} \\
U^{*} \rightarrow_{193} E^{*}=E^{*} & U^{*} \rightarrow_{194} E^{*}=E^{*} & U^{*} \rightarrow_{195} E^{*}=E^{*} \\
E^{*} \rightarrow_{193} O^{*}=O^{*} & E^{*} \rightarrow_{194} O^{*}=O^{*} & E^{*} \rightarrow_{195} O^{*}=O^{*} \\
E^{*} \rightarrow_{193} U^{*}=O^{*} & E^{*} \rightarrow_{194} U^{*}=E^{*} & E^{*} \rightarrow_{195} U^{*}=O^{*} \\
E^{*} \rightarrow_{193} E^{*}=E^{*} & E^{*} \rightarrow_{194} E^{*}=E^{*} & E^{*} \rightarrow_{195} E^{*}=E^{*} \\
O^{*} \rightarrow_{196} O^{*}=E^{*} & O^{*} \rightarrow_{197} O^{*}=E^{*} & O^{*} \rightarrow_{198} O^{*}=E^{*} \\
O^{*} \rightarrow_{196} U^{*}=E^{*} & O^{*} \rightarrow_{197} U^{*}=E^{*} & O^{*} \rightarrow_{198} U^{*}=E^{*} \\
O^{*} \rightarrow_{196} E^{*}=E^{*} & O^{*} \rightarrow_{197} E^{*}=E^{*} & O^{*} \rightarrow_{198} E^{*}=E^{*} \\
U^{*} \rightarrow_{196} O^{*}=E^{*} & U^{*} \rightarrow_{197} O^{*}=O^{*} & U^{*} \rightarrow_{198} O^{*}=O^{*} \\
U^{*} \rightarrow_{196} U^{*}=E^{*} & U^{*} \rightarrow_{197} U^{*}=O^{*} & U^{*} \rightarrow_{198} U^{*}=E^{*} \\
U^{*} \rightarrow_{196} E^{*}=E^{*} & U^{*} \rightarrow_{197} E^{*}=E^{*} & U^{*} \rightarrow_{198} E^{*}=E^{*} \\
E^{*} \rightarrow_{196} O^{*}=O^{*} & E^{*} \rightarrow_{197} O^{*}=O^{*} & E^{*} \rightarrow_{198} O^{*}=O^{*} \\
E^{*} \rightarrow_{196} U^{*}=E^{*} & E^{*} \rightarrow_{197} U^{*}=E^{*} & E^{*} \rightarrow_{198} U^{*}=E^{*} \\
E^{*} \rightarrow_{196} E^{*}=E^{*} & E^{*} \rightarrow_{197} E^{*}=E^{*} & E^{*} \rightarrow_{198} E^{*}=E^{*} \\
& &
\end{array}
$$

Four different geometrical interpretations of the element $x \in E$ in IFSs $A$ and $B$, i.e., with degrees $\mu_{A}(x)$ and $\nu_{A}(x)$, and $\mu_{B}(x)$ and $\nu_{B}(x)$; and the element $x$ from IFS $A \rightarrow_{i} B$, are shown on Fig. 2-5, where $i=193,194, \ldots, 198$. These four scenarios are related to the locations of the element $x$ in $A$ and $B$. Let us denote the element $x$ in $A$ by $x_{A}$, in $B-$ by $x_{B}$ and in $A \rightarrow_{i} B-$ by $x_{i}$ for $i=193,194, \ldots, 198$. 
Let us denote for any two elements $x, y \in E$ that $x \approx y$ if and only if $\mu_{A}(x)=\mu_{B}(y)$ and $\nu_{A}(x)=\nu_{B}(y)$.

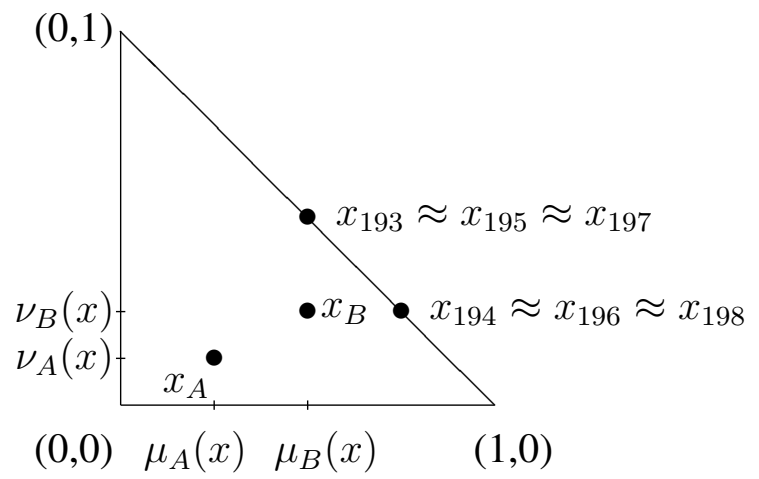

Figure 2. The geometrical interpretation - first scenario

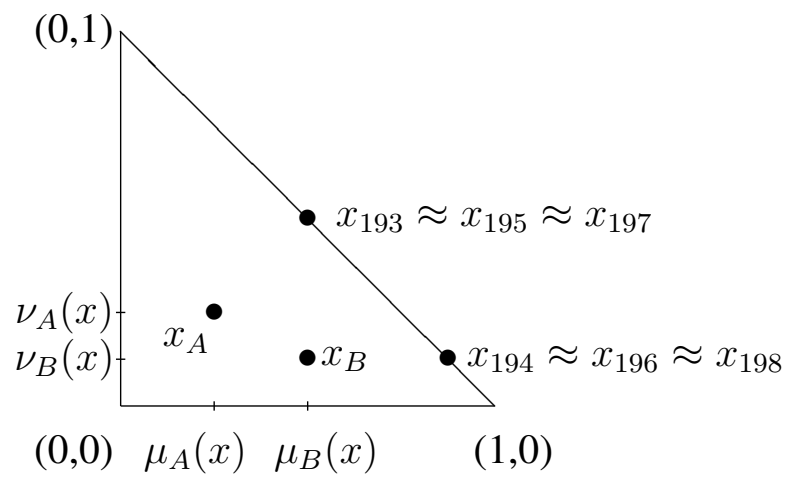

Figure 3. The geometrical interpretation - second scenario

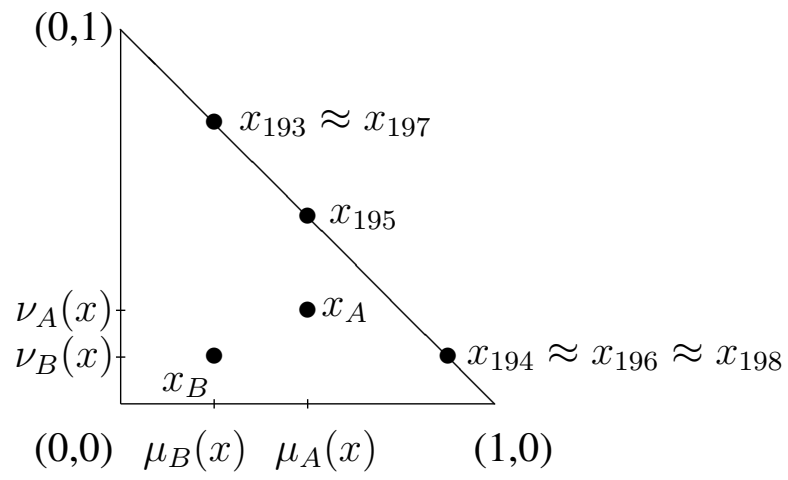

Figure 4. The geometrical interpretation - third scenario

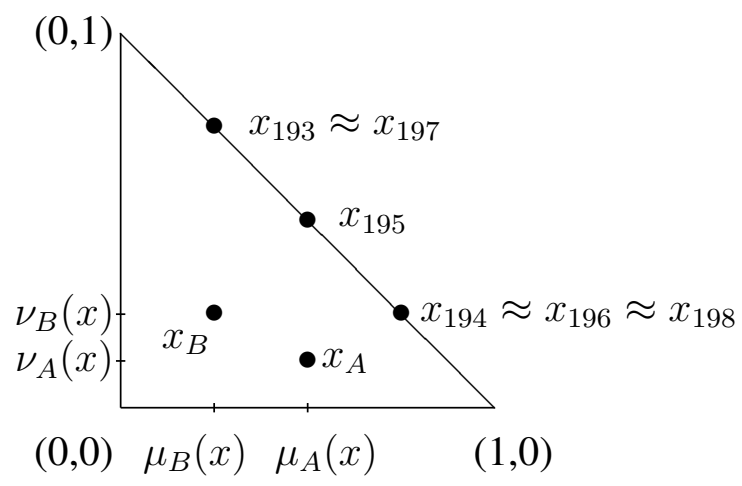

Figure 5. The geometrical interpretation - fourth scenario 
In the four examples, $x_{194} \approx x_{196} \approx x_{198}$, but as we will see in the proof of the Theorem 3.1, this is not always valid.

For every two IFSs $P$ and $Q$, let us denote $P \subseteq Q$ by $\stackrel{P}{\bullet \longrightarrow} \longrightarrow$.

Theorem 3.1. The following relations are valid:

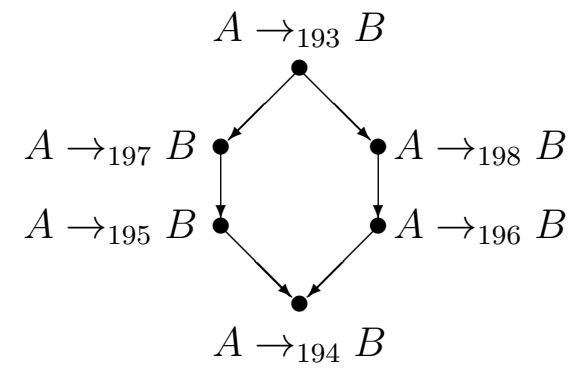

Proof: We will check sequentially the following 8 cases.

Case 1: $A \rightarrow_{195} B \subseteq A \rightarrow_{194} B$. Let

$$
X \equiv 1-\min \left(\nu_{B}(x), \max \left(\mu_{A}(x), \mu_{B}(x)\right)\right)-\max \left(\mu_{B}(x), 1-\max \left(\mu_{A}(x), \mu_{B}(x)\right)\right) .
$$

If $\mu_{A}(x) \geq \mu_{B}(x)$. Then

$$
\begin{aligned}
X & =1-\min \left(\nu_{B}(x), \mu_{A}(x)\right)-\max \left(\mu_{B}(x), 1-\mu_{A}(x)\right) \\
& =1-\min \left(\nu_{B}(x), \mu_{A}(x)\right)-1+\min \left(1-\mu_{B}(x), \mu_{A}(x)\right) \\
& =\min \left(1-\mu_{B}(x), \mu_{A}(x)\right)-\min \left(\nu_{B}(x), \mu_{A}(x)\right) \geq 0 .
\end{aligned}
$$

If $\mu_{A}(x)<\mu_{B}(x)$. Then

$$
\begin{aligned}
X & \left.=1-\min \left(\nu_{B}(x), \mu_{B}(x)\right)\right)-\max \left(\mu_{B}(x), 1-\mu_{B}(x)\right) \\
& \left.=1-\min \left(\nu_{B}(x), \mu_{B}(x)\right)\right)-1+\min \left(1-\mu_{B}(x), \mu_{B}(x)\right) \\
& =\min \left(1-\mu_{B}(x), \mu_{B}(x)\right)-\min \left(\nu_{B}(x), \mu_{B}(x)\right) \geq 0 .
\end{aligned}
$$

The fact that there are cases in which relation $\subseteq$ can be strong is shown on Fig. 6 .

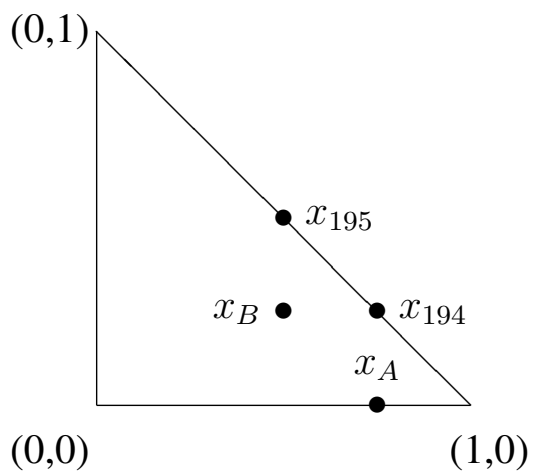

Figure 6. Example for $A \rightarrow_{195} B \subset A \rightarrow_{194} B$ 
Case 2: $A \rightarrow_{197} B \subseteq A \rightarrow_{195} B$. We see directly that

$$
\max \left(\mu_{B}(x), 1-\max \left(\mu_{A}(x), \mu_{B}(x)\right)\right)-\max \left(\mu_{B}(x), \min \left(\nu_{A}(x), 1-\mu_{B}(x)\right)\right) \geq 0
$$

because

$$
\begin{aligned}
& 1-\max \left(\mu_{A}(x), \mu_{B}(x)\right)-\min \left(\nu_{A}(x), 1-\mu_{B}(x)\right) \\
& =1-\max \left(\mu_{A}(x), \mu_{B}(x)\right)-1+\max \left(1-\nu_{A}(x), \mu_{B}(x)\right) \\
& =\max \left(1-\nu_{A}(x), \mu_{B}(x)\right)-1-\max \left(\mu_{A}(x), \mu_{B}(x)\right) \geq 0 .
\end{aligned}
$$

The fact that there are cases in which relation $\subseteq$ can be strong is shown on Fig. 7 .

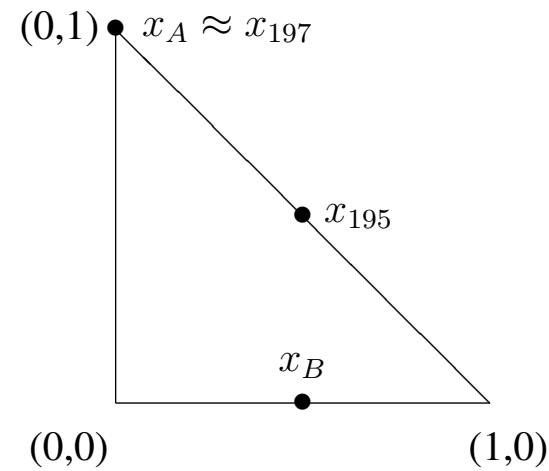

Figure 7. Example for $A \rightarrow_{197} B \subset A \rightarrow_{195} B$

Case 3: $A \rightarrow_{193} B \subseteq A \rightarrow_{197} B$. We see directly that

$$
\max \left(\mu_{B}(x), \min \left(\nu_{A}(x), 1-\mu_{B}(x)\right)\right)-\max \left(\mu_{B}(x), \min \left(\nu_{A}(x), \nu_{B}(x)\right)\right) \geq 0
$$

because

$$
\min \left(\nu_{A}(x), 1-\mu_{B}(x)\right)-\min \left(\nu_{A}(x), \nu_{B}(x)\right) \geq 0 .
$$

The fact that there are cases in which relation $\subseteq$ can be strong is shown on Fig. 8 .

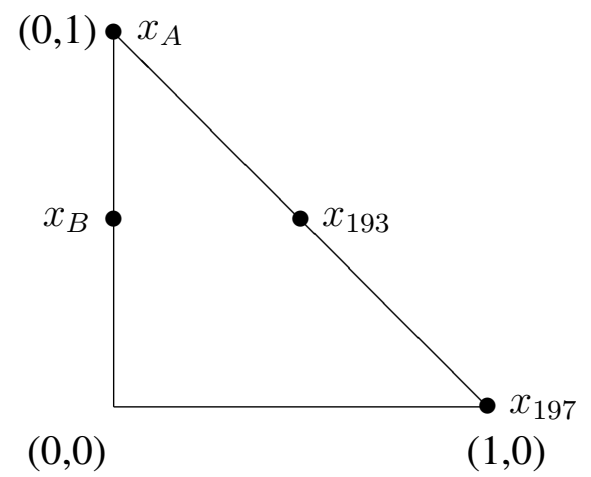

Figure 8. Example for $A \rightarrow_{193} B \subset A \rightarrow_{197} B$ 
Case 4: $A \rightarrow_{193} B \subseteq A \rightarrow_{198} B$. We see directly that

$$
\max \left(1-\nu_{B}(x), \min \left(\nu_{A}(x), \nu_{B}(x)\right)\right)-\max \left(\mu_{B}(x), \min \left(\nu_{A}(x), \nu_{B}(x)\right)\right) \geq 0
$$

because $1-\nu_{B}(x)-\mu_{B}(x) \geq 0$.

The fact that there are cases in which relation $\subseteq$ can be strong is shown on Figs. 2-5.

Case 5: $A \rightarrow_{198} B \subseteq A \rightarrow_{196} B$. We see directly that

$$
\begin{aligned}
& 1-\min \left(\nu_{B}(x), \max \left(\mu_{A}(x), 1-\nu_{B}(x)\right)\right)-\max \left(1-\nu_{B}(x), \min \left(\nu_{A}(x), \nu_{B}(x)\right)\right) \\
& =\max \left(1-\nu_{B}(x), 1-\max \left(\mu_{A}(x), 1-\nu_{B}(x)\right)\right)-\max \left(1-\nu_{B}(x), \min \left(\nu_{A}(x), \nu_{B}(x)\right)\right) \\
& =\max \left(1-\nu_{B}(x), \min \left(1-\mu_{A}(x), \nu_{B}(x)\right)\right)-\max \left(1-\nu_{B}(x), \min \left(\nu_{A}(x), \nu_{B}(x)\right)\right) \geq 0,
\end{aligned}
$$

because

$$
1-\mu_{B}(x)-\nu_{B}(x) \geq 0 .
$$

The fact that there are cases in which relation $\subseteq$ can be strong is shown on Fig. 9 .

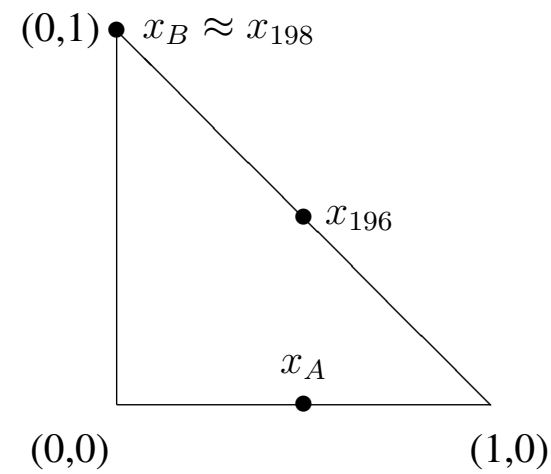

Figure 9. Example for $A \rightarrow_{198} B \subset A \rightarrow_{196} B$

Case 6: $A \rightarrow_{196} B \subseteq A \rightarrow_{194} B$. We see directly that

$$
\begin{aligned}
& 1-\min \left(\nu_{B}(x), \max \left(\mu_{A}(x), \mu_{B}(x)\right)\right)-1+\min \left(\nu_{B}(x), \max \left(\mu_{A}(x), 1-\nu_{B}(x)\right)\right) \\
& =\min \left(\nu_{B}(x), \max \left(\mu_{A}(x), 1-\nu_{B}(x)\right)\right)-\min \left(\nu_{B}(x), \max \left(\mu_{A}(x), \mu_{B}(x)\right)\right) \geq 0,
\end{aligned}
$$

because

$$
\max \left(\mu_{A}(x), 1-\nu_{B}(x)\right)-\max \left(\mu_{A}(x), \mu_{B}(x)\right) \geq 0 .
$$

The fact that there are cases in which relation $\subseteq$ can be strong is shown on Fig. 10 .

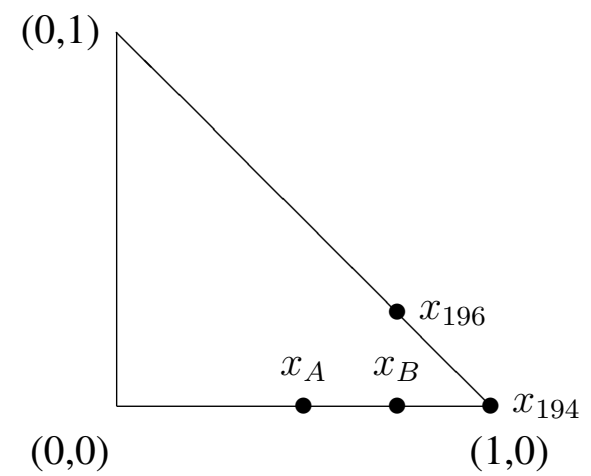

Figure 10. Example for $A \rightarrow_{196} B \subset A \rightarrow_{194} B$ 
Case 7: The IFSs $A \rightarrow_{195} B$ and $A \rightarrow_{196} B$ are independent. Really, let

$$
X \equiv 1-\min \left(\nu_{B}(x), \max \left(\mu_{A}(x), 1-\nu_{B}(x)\right)\right)-\max \left(\mu_{B}(x), 1-\max \left(\mu_{A}(x), \mu_{B}(x)\right)\right) .
$$

Then we obtain that

$$
\begin{aligned}
X & =1-\min \left(\nu_{B}(x), \max \left(\mu_{A}(x), 1-\nu_{B}(x)\right)\right)-1+\min \left(1-\mu_{B}(x), \max \left(\mu_{A}(x), \mu_{B}(x)\right)\right) \\
& =\min \left(1-\mu_{B}(x), \max \left(\mu_{A}(x), \mu_{B}(x)\right)\right)-\min \left(\nu_{B}(x), \max \left(\mu_{A}(x), 1-\nu_{B}(x)\right)\right) .
\end{aligned}
$$

If $\mu_{A}(x)<\mu_{B}(x) \leq 1-\nu_{B}(x)$, then

$$
X=\min \left(1-\mu_{B}(x), \mu_{B}(x)\right)-\min \left(\nu_{B}(x), 1-\nu_{B}(x)\right) .
$$

For example, if $\mu_{B}(x)=\frac{1}{3}$ and $\nu_{B}(x)=\frac{1}{4}$, then

$$
X=\min \left(1-\frac{1}{3}, \frac{1}{3}\right)-\min \left(\frac{1}{4}, 1-\frac{1}{4}\right)=\frac{1}{3}-\frac{1}{4}>0
$$

but if $\mu_{B}(x)=\frac{1}{4}$ and $\nu_{B}(x)=\frac{1}{3}$, then

$$
X=\min \left(1-\frac{1}{4}, \frac{1}{4}\right)-\min \left(\frac{1}{3}, 1-\frac{1}{3}\right)=\frac{1}{4}-\frac{1}{3}<0 .
$$

Therefore, the IFSs $A \rightarrow_{195} B$ and $A \rightarrow_{196} B$ are independent.

Case 8: The IFSs $A \rightarrow_{197} B$ and $A \rightarrow_{198} B$ are independent. Really, let

$$
X \equiv \max \left(\mu_{B}(x), \min \left(\nu_{A}(x), 1-\mu_{B}(x)\right)\right)-\max \left(1-\nu_{B}(x), \min \left(\nu_{A}(x), \nu_{B}(x)\right)\right) .
$$

If $\nu_{A}(x)>1-\mu_{B}(x) \geq \nu_{B}(x)$, then

As in the previous case, we see that if $\mu_{B}(x)=\frac{1}{3}$ and $\nu_{B}(x)=\frac{1}{4}$, then

$$
X=\max \left(\frac{1}{3}, 1-\frac{1}{3}\right)-\max \left(1-\frac{1}{4}, \frac{1}{4}\right)=\frac{2}{3}-\frac{3}{4}<0,
$$

while, if $\mu_{B}(x)=\frac{1}{4}$ and $\nu_{B}(x)=\frac{1}{3}$, then

$$
X=\max \left(\frac{1}{4}, 1-\frac{1}{4}\right)-\max \left(1-\frac{1}{3}, \frac{1}{3}\right)=\frac{3}{4}-\frac{2}{3}>0 .
$$

Therefore, the IFSs $A \rightarrow_{197} B$ and $A \rightarrow_{198} B$ are independent.

Using the well-known formula (see, e.g., [25])

$$
\neg p=p \rightarrow 0,
$$

we see that

$$
\begin{aligned}
\neg^{1} A & =\neg^{5} A=\neg^{6} A=\left\{\left\langle x, \nu_{A}(x), 1-\nu_{A}(x)\right\rangle \mid x \in E\right\}, \\
\neg^{2} A & =\neg^{3} A=\neg^{4} A=\left\{\left\langle x, 1-\mu_{A}(x), \mu_{A}(x)\right\rangle \mid x \in E\right\} .
\end{aligned}
$$

Therefore,

$$
\neg^{1} A=\neg^{5} A=\neg^{6} A \subseteq \neg^{2} A=\neg^{3} A=\neg^{4} A .
$$

We must mention that the first negation in the present paper coincides with negation $\neg_{4}$ and the second one coincides with $\urcorner_{8}$ from [9]. 
Nine axioms for implications are introduced in [24]. They are the following:

Axiom 1. $(\forall x, y)(x \leq y \rightarrow(\forall z)(I(x, z) \geq I(y, z)))$.

Axiom 2. $(\forall x, y)(x \leq y \rightarrow(\forall z)(I(z, x) \leq I(z, y)))$.

Axiom 3. $(\forall y)(I(0, y)=1)$.

Axiom 4. $(\forall y)(I(1, y)=y)$.

Axiom 5. $(\forall x)(I(x, x)=1)$.

Axiom 6. $(\forall x, y, z)(I(x, I(y, z))=I(y, I(x, z)))$.

Axiom 7. $(\forall x, y)(I(x, y)=1$ iff $x \leq y)$.

Axiom 8. $(\forall x, y)(I(x, y)=I(N(y), N(x)))$, where $N$ is an operation for a negation.

Axiom 9. $I$ is a continuous function.

Following [8], we will mention that if the axiom is valid as an Intuitionistic Fuzzy Tautology (IFT), the number of the axiom is marked with an asterisk $(*)$. These axioms are:

Axiom 3*. $(\forall y)(I(0, y)$ is an IFT).

Axiom 5*. $(\forall x)(I(x, x)$ is an IFT).

Axiom $7 * .(\forall x, y)(I(x, y)$ is an IFT iff $x \leq y)$.

Theorem 3.2. All implications satisfy only Axioms 1 and 9 as tautologies; implications $\rightarrow_{193}$, $\rightarrow_{195}, \rightarrow_{197}$ satisfy Axiom 6 as tautologies.

Proof: Let the three IFSs $A, B$ and $C$ be given and let $A \subseteq B$. Therefore, for each $x \in E$ :

$$
\mu_{A}(x) \leq \mu_{B}(x), \quad \nu_{A}(x) \geq \nu_{B}(x) .
$$

We construct

$A \rightarrow{ }_{193} C=\left\{\left\langle x, \max \left(\mu_{C}(x), \min \left(\nu_{A}(x), \nu_{C}(x)\right)\right), 1-\max \left(\mu_{C}(x), \min \left(\nu_{A}(x), \nu_{C}(x)\right)\right)\right\rangle \mid x \in E\right\}$

and

$B \rightarrow{ }_{193} C=\left\{\left\langle x, \max \left(\mu_{C}(x), \min \left(\nu_{B}(x), \nu_{C}(x)\right)\right), 1-\max \left(\mu_{C}(x), \min \left(\nu_{B}(x), \nu_{C}(x)\right)\right)\right\rangle \mid x \in E\right\}$.

Hence, by analogy with the above proof, we check

$$
\max \left(\mu_{C}(x), \min \left(\nu_{A}(x), \nu_{C}(x)\right)\right)-\max \left(\mu_{C}(x), \min \left(\nu_{B}(x), \nu_{C}(x)\right)\right) \geq 0,
$$

that proves the Theorem in the case of implication $\rightarrow_{193}$.

Axiom 9 is obviously true, because functions max and min are continuous. The rest assertions are proved by the same manner.

For all of the rest axioms, counterexamples exist.

The proofs of the next assertions are made in the same manner.

Theorem 3.3. As IFTs, all implications satisfy Axiom 1, implications $\rightarrow_{194}, \rightarrow_{195}, \ldots, \rightarrow_{198}$ satisfy Axiom 4, implications $\rightarrow_{193}, \rightarrow_{195}, \rightarrow_{197}$ satisfy Axiom 6 and implications $\rightarrow_{194}, \rightarrow_{195}$, $\rightarrow_{196}, \rightarrow_{198}$ satisfy Axiom 8. 
Following [26], we give the axioms of the intuitionistic logic.
(a) $A \rightarrow A$,
(b) $A \rightarrow(B \rightarrow A)$,
(c) $A \rightarrow(B \rightarrow(A \cap B))$,
(d) $(A \rightarrow(B \rightarrow C)) \rightarrow(B \rightarrow(A \rightarrow C))$,
(e) $(A \rightarrow(B \rightarrow C)) \rightarrow((A \rightarrow B) \rightarrow(A \rightarrow C))$,
(f) $A \rightarrow \neg \neg A$,
(g) $\neg(A \cap \neg A)$,
(h) $(\neg A \cup B) \rightarrow(A \rightarrow B)$,
(i) $\neg(A \cup B) \rightarrow(\neg A \cap \neg B)$,
(j) $(\neg A \cap \neg B) \rightarrow \neg(A \cup B)$,
(k) $(\neg A \cup \neg B) \rightarrow \neg(A \cap B)$,
(l) $(A \rightarrow B) \rightarrow(\neg B \rightarrow \neg A)$,
(m) $(A \rightarrow \neg B) \rightarrow(B \rightarrow \neg A)$,
(n) $\neg \neg \neg A \rightarrow \neg A$,
(o) $\neg A \rightarrow \neg \neg \neg A$,
(p) $\neg \neg(A \rightarrow B) \rightarrow(A \rightarrow \neg \neg B)$,
(q) $(C \rightarrow A) \rightarrow((C \rightarrow(A \rightarrow B)) \rightarrow(C \rightarrow B))$.

Theorem 3.4. None of the axioms $(a)-(q)$ is valid as a tautological set for the six new implications.

Theorem 3.5. For every three IFSs $A, B$ and $C$ :

(a) Implication $\rightarrow_{193}$ satisfies Axioms 6, 7, 9, 10, 11, 12, 13, 14, 15, 16.

(b) Implications $\rightarrow_{194}$ and $\rightarrow_{196}$ satisfy Axioms 1, 2, 3, 4, 6, 7, 8, 9, 10, 11, 13, 14, 15.

(c) Implications $\rightarrow_{195}$ and $\rightarrow_{198}$ satisfy all of the axioms.

(d) Implication $\rightarrow_{197}$ satisfies Axioms 4, 5, 6, 7, 9, 10, 11, 12, 13, 14, 15, 16, 17 as IFTSs.

The proofs of these theorems are similar to the above ones.

\section{Conclusion}

As it is shown in [1, 2, 3], for each intuitionistic fuzzy implication, one or three intuitionistic fuzzy disjunctions and conjunctions are introduced. For example, for disjunction, the following formulas are used:

$$
\begin{aligned}
& p \vee_{1} q=\neg p \rightarrow q, \\
& p \vee_{2} q=\neg p \rightarrow \neg \neg q,
\end{aligned}
$$


where operation intuitionistic fuzzy negation $(\neg)$ is generated by the respective intuitionistic fuzzy implication $(\rightarrow)$; and

$$
p \vee_{3} q=\neg p \rightarrow q,
$$

where the negation $\neg$ is the classical one.

In future, the respective intuitionistic fuzzy disjunctions and conjunctions, associated to the six new implications will be introduced and their properties will be studied. Having in mind the form of the negations, generated by the new implications, we must immediately mention that each of these implications will generate three different disjunctions and conjunctions (cf. [1, 2, 3]).

\section{Acknowledgement}

This research was funded by Bulgarian National Science Fund, grant number KP-06-N22/1/2018 "Theoretical research and applications of InterCriteria Analysis".

\section{References}

[1] Angelova, N., \& Stoenchev, M. (2015/2016). Intuitionistic fuzzy conjunctions and disjunctions from first type. Annual of "Informatics" Section, Union of Scientists in Bulgaria, 8, 1-17.

[2] Angelova, N., Stoenchev, M., \& Todorov, V. (2017). Intuitionistic fuzzy conjunctions and disjunctions from second type. Issues in Intuitionistic Fuzzy Sets and Generalized Nets, 13, 143-170.

[3] Angelova, N., \& Stoenchev, M. (2017). Intuitionistic fuzzy conjunctions and disjunctions from third type. Notes on Intuitionistic Fuzzy Sets, 23(5), 29-41.

[4] Atanassov, K. (1999). Intuitionistic Fuzzy Sets: Theory and Applications, Springer, Heidelberg.

[5] Atanassov, K. (2006). On some intuitionistic fuzzy implication. Comptes Rendus de l'Academie bulgare des Sciences, 59(1), 21-26.

[6] Atanassov, K. (2006). A new intuitionistic fuzzy implication from a modal type. Advanced Studies in Contemporary Mathematics, 12(1), 117-122.

[7] Atanassov, K. (2011). Second Zadeh's intuitionistic fuzzy implication. Notes on Intuitionistic Fuzzy Sets, 17(3), 11-14.

[8] Atanassov, K. (2012). On Intuitionistic Fuzzy Sets Theory, Springer, Berlin.

[9] Atanassov, K. (2017). Intuitionistic Fuzzy Logics, Springer, Cham.

[10] Atanassov, K. (2019) On the intuitionistic fuzzy implication $\rightarrow_{191}$. Notes on Intuitionistic Fuzzy Sets, 25(4), 1-6. 
[11] Atanassov, K., Angelova, N., \& Atanassova, V. (2021). On an Intuitionistic Fuzzy Form of the Goguen's Implication. Mathematics 9(6), Article ID 676.

[12] Atanassov, K. (2021). Third Zadeh's Intuitionistic Fuzzy Implication. Mathematics, 9(6), Article ID 619.

[13] Atanassova, L. (2009). A new intuitionistic fuzzy implication. Cybernetics and Information Technologies, 9(2), 21-25.

[14] Atanassova, L. (2009). On some properties of intuitionistic fuzzy negation $\neg @$. Notes on Intuitionistic Fuzzy Sets, 15(1), 32-35.

[15] Atanassova, L. (2012). On two modifications of the intuitionistic fuzzy implication $\rightarrow @$. Notes on Intuitionistic Fuzzy Sets, 18(2), 26-30.

[16] Atanassova, L. (2013). On the modal form of the intuitionistic fuzzy implications $\rightarrow \rightarrow_{@}^{\prime}$ and $\rightarrow{ }_{@}^{\prime \prime}$. Issues in Intuitionistic Fuzzy Sets and Generalized Nets, 10, 5-11.

[17] Atanassova, L. (2015). Remark on Dworniczak's intuitionistic fuzzy implications. Part 1. Notes on Intuitionistic Fuzzy Sets, 21(3), 18-23.

[18] Atanassova, L. (2015/2016). Remark on Dworniczak's intuitionistic fuzzy implications. Part 2. Issues in Intuitionistic Fuzzy Sets and Generalized Nets, 12, 61-67.

[19] Chen, J., \& Kundu, S. (1996). A sound and complete fuzzy logic system using Zadeh's implication operator. Lecture Notes in Computer Science, 1079, 233-242.

[20] Dworniczak, P. (2010). Some remarks about the L. Atanassova's paper "A new intuitionistic fuzzy implication”. Cybernetics and Information Technologies, 10(3), 3-9.

[21] Dworniczak, P. (2010). On one class of intuitionistic fuzzy implications. Cybernetics and Information Technologies, 10(4), 13-21.

[22] Dworniczak, P. (2011). On some two-parametric intuitionistic fuzzy implications. Notes on Intuitionistic Fuzzy Sets, 17(2), 8-16.

[23] Feys, R. (1965). Modal logics, Gauthier-Villars, Paris.

[24] Klir, G., \& Yuan, B. (1995). Fuzzy Sets and Fuzzy Logic, Prentice Hall, New Jersey.

[25] Mendelson, E. (1964). Introduction to Mathematical Logic, D. Van Nostrand, Princeton, New Jersey.

[26] Rasiova, H., \& Sikorski, R.(1963). The Mathematics of Metamathematics, Pol. Acad. of Sci., Warszawa.

[27] Vassilev, P., \& Atanassov, K. (2019). Extensions and Modifications of Intuitionistic Fuzzy Sets, "Prof. Marin Drinov" Academic Publishing House, Sofia. 